Mon. Not. R. Astron. Soc. 000, 000-000 (0000) Printed October 25, $2018 \quad$ (MN $2 \mathrm{TT}_{\mathrm{E}} \mathrm{X}$ style file v2.2)

\title{
A Candidate Dual AGN in a Double-peaked Emission-line Galaxy with Precessing Radio Jets
}

\author{
Rubinur, K. ${ }^{1,2 \star}$, M. Das ${ }^{1}$, P. Kharb ${ }^{3}$, M. Honey ${ }^{1,2}$ \\ ${ }^{1}$ Indian Institute of Astrophysics, Bangalore 560034, India \\ ${ }^{2}$ Pondicherry University, R. Venkataraman Nagar, Kalapet, 605014 Pondicherry, India \\ ${ }^{3}$ National Centre for Radio Astrophysics - Tata Institute of Fundamental Research, \\ Pune University Campus, Post Bag 3, Ganeshkhind Pune 411007, India
}

October 25, 2018

\begin{abstract}
We present high resolution radio continuum observations with the Karl G. Jansky Very Large Array at $6,8.5,11.5$ and $15 \mathrm{GHz}$ of the double-peaked emission-line galaxy 2MASXJ12032061+1319316. The radio emission has a prominent S-shaped morphology with highly symmetric radio jets that extend over a distance of $\sim 1.5^{\prime \prime}(1.74 \mathrm{kpc})$ on either side of the core of size $\sim 0.1^{\prime \prime}(116 \mathrm{pc})$. The radio jets have a helical structure resembling the precessing jets in the galaxy NGC 326 which has confirmed dual active galactic nuclei (AGN). The nuclear bulge velocity dispersion gives an upper limit of $(1.56 \pm 0.26) \times 10^{8} \mathrm{M}_{\odot}$ for the total mass of nuclear black hole(s). We present a simple model of precessing jets in 2MASXJ1203 and find that the precession timescale is around $10^{5}$ years: this matches the source lifetime estimate via spectral aging. We find that the expected super massive black hole $(\mathrm{SMBH})$ separation corresponding to this timescale is $0.02 \mathrm{pc}$. We used the double peaked emission lines in 2MASXJ1203 to determine an orbital speed for a dual AGN system and the associated jet precession timescale, which turns out to be more than the Hubble time, making it unfeasible. We conclude that the S-shaped radio jets are due to jet precession caused either by a binary/dual SMBH system, a single SMBH with a tilted accretion disk or a dual AGN system where a close pass of the secondary SMBH in the past has given rise to jet precession.
\end{abstract}

Key words: galaxies: active, galaxies: individual (2MASXJ12032061+1319316), galaxies: Seyfert, galaxies: jets, radio continuum: galaxies

\section{INTRODUCTION}

According to the $\Lambda \mathrm{CDM}$ Universe, massive galaxies form through the mergers of smaller galaxies (Springel 2005). During mergers, the super massive black holes (SMBHs) of the individual galaxies sink to the center of the merger remnant (Begelman, Blandford \& Rees 1980) and form a SMBH pair. Simulations show that mergers are accompanied by massive gas inflows (Hopkins \& Hernquist 2009). This can result in accretion of gas onto the SMBHs resulting in dual active galactic nuclei (DAGN) with SMBH separations of $0.1-10 \mathrm{kpc}$ or binary AGN with separations $<100 \mathrm{pc}$ (BurkeSpolaor 2015). The gas accretion may also give rise to radio jets that launch along the direction of black hole spin (Rees et al. 1982). The binary SMBHs will eventually coalesce producing huge amount of gravitational waves (Thorne \& Braginskii 1976). Theoretical studies predict that DAGN

* E-mail: rubinur@iiap.res.in should be common, but the number of resolved DAGN is low mainly because the required resolution is very hard to achieve. To date, there are only $\sim 23$ resolved DAGN (Deane et al. 2014, Müller-Sánchez et al. 2015).

The resolved DAGN are detected through high resolution imaging at radio, X-ray, optical or Near infrared (NIR) wavelengths. There is only one confirmed binary AGN which is the radio galaxy $0402+379$. In this case, the Very Long Baseline Array (VLBA) has resolved the binary at 7 pc separation (Rodriguez et al. 2006). On kpc scales, the few convincing examples are LBQS 0103-2753 (Junkkarinen et al. 2001), NGC 6240 (Komossa et al. 2003), 3C 75 (Hudson et al. 2006), Mrk 463 (Bianchi et al.|2008), Mrk 739 (Koss et al. 2012). More examples are in Fu et al. (2011a); Liu et al. (2013); Comerford et al. (2015). In optical and NIR, the detection of two cores in imaging is not sufficient because one of the cores can be a starburst nucleus or a stellar bulge. Therefore, spectroscopic observations of the cores are required along with imaging (McGurk et al. 2015). 
There are a few indirect signatures of DAGN/binary AGN: (1) Periodicity in optical variability; (2) Doublepeaked AGN (DPAGN) emission lines in optical nuclear spectra; (3) S- or X-shaped radio morphology. The initial detections were serendipitous and the earliest DAGN were detected from their optical variability (e.g. OJ287 Sillanpaa et al. 1988; Lehto \& Valtonen 1996). Recently a close supermassive black-hole binary in quasar PG 1302-102 has been discovered from optical periodicity (Graham et al. 2015). The other example is PSO J334.2028+01.4075 (Liu et al. 2015).

DPAGN are one of the signature of DAGN (Zhou et al. 2004). Since the advent of high resolution spectroscopic surveys (e.g. SDSS) $)^{1}$ large samples of candidate DAGN have been identified from DPAGN spectra (Liu et al. 2010). But DPAGN can also be due to rotating disks (Greene \& Ho 2005: Müller-Sánchez et al. 2011; Kharb et al. |2015), jetISM interaction or outflows (Whittle et al. 2005, Rosario et al. 2010). Therefore, in order to confirm dual AGN in DPAGN galaxies, one has to carry out high resolution imaging at different wavelengths. At present, almost 30\% confirmed DAGNs are from DPAGN samples (e.g., Fu et al. 2011a McGurk et al. 2011). Tingay \& Wayth (2011) observed 11 DPAGN using VLBI but did not find any double radio cores. They concluded that DPAGN may not be a good indicator of dual AGN. However, McGurk et al. (2015) have pointed out that the contribution of DPAGN sample in identifying DAGN is significant. In recent studies, additional observations - such as long-slit spectroscopy, have been used for detecting DAGN in high resolution radio imaging observations (Comerford et al. 2012 Müller-Sánchez et al. 2015).

The presence of S- or X-shaped radio jets was suggested to be connected with binary SMBHs by Begelman, Blandford \& Rees (1980). Merger of two SMBHs can give rise to inversion symmetry in radio jets ( $\mathrm{S}, \mathrm{Z}$ and $\mathrm{X}$ shaped sources) (Rottmann 2001; Komossa 2006, Gergely \& Biermann 2009. Mezcua et al. 2011). Jet axis reorientation in the presence of a companion galaxy can be the reason for peculiar radio morphologies (Wirth, Smarr \& Gallagher 1982). The interaction between the DAGN can result in precessing jets that appear as X- or S-shaped radio sources (Merritt \& Ekers 2002, Zier \& Biermann 2002). Ekers et al. (1978) observed NGC 326 which is a Z-shaped radio source and explained the morphology by jet precession. NGC 326 is now a confirmed dual AGN system (Murgia et al. 2001, Hodges-Kluck \& Reynolds 2012). Another example is the micro-quasar SS433. This is a famous S-shaped radio source and it is a binary system (Blundell \& Bowler 2004). Gopal-Krishna, Biermann \& Wiita (2003) have suggested that Z- or S- shaped sources are SMBHs that are close to coalescence and are associated with gravitational radiation, whereas $\mathrm{X}$-shaped radio sources are merged systems and have radiated away gravitational waves. But warping of the accretion disks (Pringle 1996) or back flowing gas (Leahy \& Williams 1984) can also produce some signature of X- or S- shaped galaxies.

We find evidence of S-shaped radio jets in the doublepeaked AGN of 2MASXJ12032061+1319316 (2MASXJ1203 hereafter). In Section 2 we describe the target galaxy. The observations and data reduction are described in Sections 3

1 www.sdss.org and 4 In Section 5.1 we describe the results of our 6, 8.5, 11.5 and $15 \mathrm{GHz}$ Expanded Very Large Array (EVLA) observations. 2D composition of galaxy image is described in Section 5.2. Further estimation of SMBH mass and other parameters are discussed in Sections 5.3. In Section 5.4 we describe the spectral index map. We also present a simple model of the precessing jets and a rough estimate of the precession timescale in Section 5.5 In Section 5.6, we estimate the minimum magnetic field and lifetime of the source from the equipartition theorem. In Section 6 we discuss the origin of the S-shaped morphology and DPAGN emission from 2MASXJ1203. We summarize our conclusions in Section 7. Throughout this paper we assume a value of $\Omega_{m}=0.27$, and $H_{0}=73.0 \mathrm{~km} \mathrm{~s}^{-1} \mathrm{Mpc}^{-1}$. The spectral index, $\alpha$, is defined such that the flux density at frequency $\nu$ is $\mathrm{S}_{\alpha} \propto \nu^{\alpha}$.

\section{THE TARGET GALAXY}

The galaxy 2MASXJ1203 is part of a larger ongoing study of DPAGN at $6 \mathrm{GHz}$ using the EVLA (Project ID: VLA/15A068) (Rubinur K. et al. 2016B in preparation). Our preliminary data analysis at $6 \mathrm{GHz}$ revealed that the nuclear emission had an interesting S-shaped morphology and two hotspots. On analysing additional archival data at higher frequencies ( 8.5 and $11.5 \mathrm{GHz}$ ), we clearly resolved the compact core and found S-shaped radio jets. 2MASXJ1203 has been studied earlier at optical wavelengths in DPAGN surveys (Fu et al. 2012, Ge et al. 2012, Wang et al. 2009). The galaxy has a moderate redshift $(\mathrm{z}=0.058)$, a compact core and extended disk (Table 1). It may be an S0 or spiral galaxy (Fathi et al. 2010) but its HyperLeda ${ }^{2}$ classification has a large error (Makarov et al. 2014). In Section 5.2 we discuss our own 2D galaxy image decomposition using GALFIT. The optical spectrum shows double peaks in [O III], [O II], $\mathrm{H} \alpha$, [N II] emission lines (Figure 1). The underlying stellar velocity dispersion is $189.9 \mathrm{~km} \mathrm{sec}^{-1}$ (see Section 4). The nucleus shows radio emission in both NRAO VLA Sky Survey (NVSS) ${ }^{3}$ and Faint Images of the Radio Sky at Twenty-cm (FIRST) 4 images at $1.4 \mathrm{GHz}$. The NVSS peak flux density is $0.108 \mathrm{Jy}$ at $1.4 \mathrm{GHz}$. The nucleus has a Seyfert 2 classification and is radio-loud with a radio loudness parameter of 156 (Fu et al. 2012). The Rossi All Sky Survey (RASS) ${ }^{5}$ covered the source region during one of its survey scans for about $440 \mathrm{~s}$; but 2MASXJ1203 is not detected in the RASS faint source catalogue which has a flux limit of $F_{x} \leqslant 1 \times 10^{-13}$ erg $\mathrm{cm}^{-2} \mathrm{~s}^{-1}$. The galaxy was observed by GALEX Survey for 107s in the near ultraviolet (NUV) band; the NUV flux density is $9.60 \times 10^{-6} \mathrm{Jy}$. NASA/IPAC Extragalactic Database $(\mathrm{NED})^{6}$ shows that there are two galaxies within the velocity range from $17015 \mathrm{~km} \mathrm{~s}^{-1}$ to $18015 \mathrm{~km} \mathrm{~s}^{-1}$. However, the galaxy does not show any signatures of interaction such as tidal tails in its optical image and hence may not be undergoing a major merger. A minor merger, however, cannot be ruled out.

\footnotetext{
2 http://leda.univ-lyon1.fr/

3 http://www.cv.nrao.edu/nvss/

4 http://www.cv.nrao.edu/first/

5 https://heasarc.gsfc.nasa.gov/docs/xte/XTE.html

6 http://ned.ipac.caltech.edu/
} 


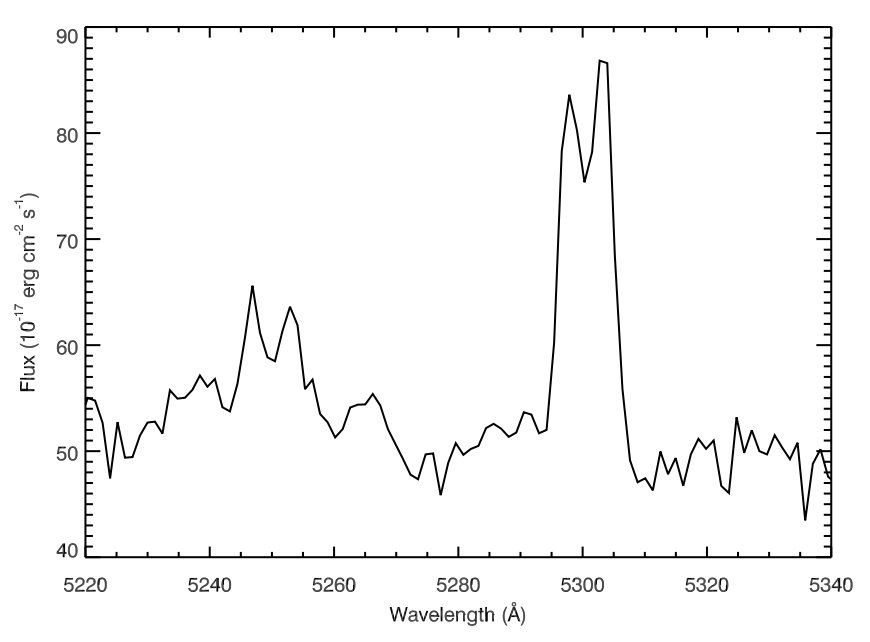

Figure 1. The double peaked emission lines in the SDSS optical spectrum from the nuclear region of SDSSJ120320.7+131931. The lines are [OIII, $\lambda 5007 \AA]$ and [OIII $\lambda 4959 \AA]$ of the [O III] doublet. There is no broadening which suggests the absence of AGN outflows.

\section{OBSERVATION AND ARCHIVAL DATA}

We observed 2MASXJ1203 on 20 July 2015 (Project ID: 15A-068) (Table 2p in the C-band at $6 \mathrm{GHz}$ using the A configuration (resolution $0.33^{\prime \prime}$ ) for 5 minutes along with the flux calibrator, J0542+4951 for 10 minutes. The nearest phase calibrator, J1239+0730 was observed for 50 seconds. The observations were done with a $1792 \mathrm{MHz}$ wide baseband centered at $5.935 \mathrm{GHz}$ with fourteen spectral windows, each of which have 64 channels with frequency resolution of $2 \mathrm{MHz}$. We also reduced the $8.5 \mathrm{GHz}$ and $11.5 \mathrm{GHz}$ archival data of 2MASXJ1203 (Project ID:VLA/13B-020). The observations were carried out in the X-band, with two frequency centers at $8.5 \mathrm{GHz}$ and $11.5 \mathrm{GHz}$ in the A configuration with eight spectral windows. We have carried out EVLA $15 \mathrm{GHz}$ observations of 2MASXJ1203 in the B array configuration (resolution $0.42^{\prime \prime}$ ) (Project ID: 16A-144) on 29th May 2016. 2MASX1203 was observed for 16 minutes along with the flux calibrator 3C 286 and phase calibrator J1224+2122. 3C 286 was observed for 11 minutes and J1224+2122 was observed for 8 minutes.

\section{DATA REDUCTION}

We have used the Common Astronomy Software Applications (CASA) (McMullin et al. 2007) and Astronomical Image Processing System (AIPS) packages for data reduction. The bad data was flagged to obtain good solutions. We used PLOTMS to identify the bad data and the task FLAGDATA to flag it. We have used the task GAINCAL to obtain the calibration solutions and applied it using the task APPLYCAL. After a satisfactory calibration we imaged the source using the task CLEAN. We made both natural and uniform weighted images using the Briggs robust parameter 0.5 and -0.5 respectively. The natural weighted images recovered most of the flux density but gave a poor spatial resolution; hence we used it to derive the total extent of the radio jets.

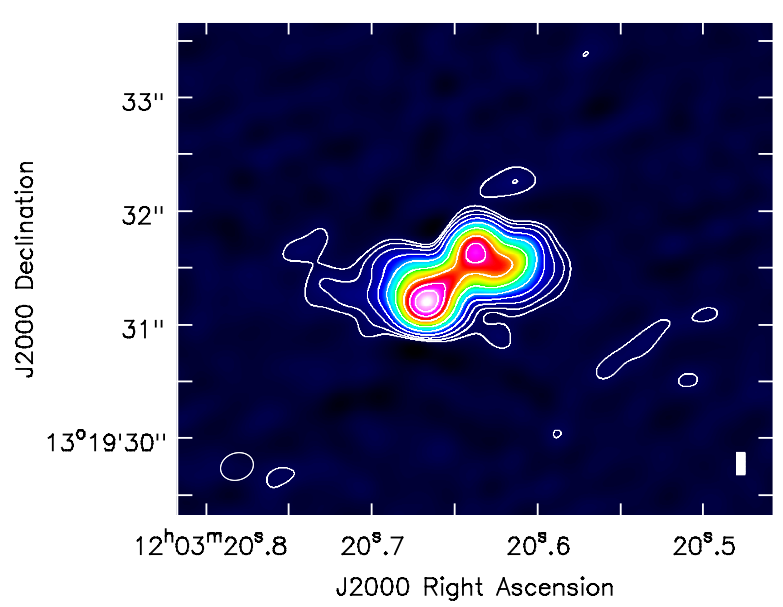

Figure 2. The uniform weighted $6 \mathrm{GHz}$ EVLA image of 2MASXJ12032061+131931. The beam size is $0.29^{\prime \prime} \times 0.24^{\prime \prime}$. The rms noise in the image is $\sim 20 \mu \mathrm{Jy}$. The contour levels correspond to $0.60,1.25,2.5,5,10,20,40,60,80 \%$ of peak flux density at $11.90 \mathrm{mJy}$.

The uniform weighted maps gave a better spatial resolution that enabled us to distinguish between the core and the jets. For the $6,8.5$ and $15 \mathrm{GHz}$ maps, we obtained good images after one round of phase self-calibration. For $11.5 \mathrm{GHz}$, we obtained a better image after two runs of phase selfcalibration. We have used IMFIT and IMSTAT tasks to get the core size, peak flux density and noise (Table 3 .

We have used the $8.5 \mathrm{GHz}$ and $11.5 \mathrm{GHz}$ images to generate the spectral index map (Figure 8). We made a new $11.5 \mathrm{GHz}$ image with same restoring beam as the $8.5 \mathrm{GHz}$ map by constraining the beam size in the task CLEAN. We used the task COMB in AIPS and task IMMATH in CASA to obtain the spectral index map and spectral index error map. The spectral index image was created after blanking flux density values below $3 \sigma$, at both frequencies. We followed the similar procedure to make spectral index map with $6 \mathrm{GHz}$ and $15 \mathrm{GHz}$ images (Figure 9p.

We have used the SDSS DR 12 I-band image for checking the morphology of 2MASXJ1203 using GALFIT (Peng et al. 2010, 2002). The SDSS images were first converted back into count units using a IDL program. We created the Point Spread Function (PSF) and masked the near by bright sources in the frame to get an accurate fitting. The residual images are produced in GALFIT by subtracting the image made by the convolution of the model with PSF from the original galactic image.

We have used the pPXF (Penalized Pixel-Fitting stellar kinematics extraction) code (Cappellari \& Emsellem $2004)$ to calculate the velocity dispersion of the underlying stellar population in the nucelar region, which uses the Gauss-Hermite parametrization technique (Gerhard 1993. We have used the SDSS DR12 spectrum for this task (Figure 1). The code first masks the emission lines in the spectrum and then fits a model to the absorption lines using a combination of single stellar population templates of different ages. It starts with initial guess values of the galaxy systemic velocity and stellar velocity dispersion $(\sigma)$. The 


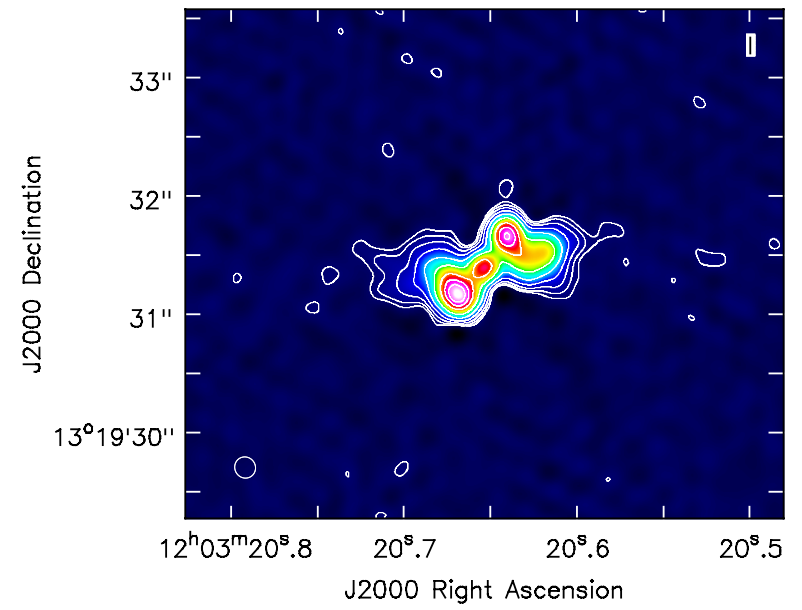

Figure 3. The uniform weighted $8.5 \mathrm{GHz}$ EVLA image of 2MASXJ12032061+131931. The beam size is $0.18^{\prime \prime} \times 0.17^{\prime \prime}$. The contour levels correspond to $0.60,1.25,2.5,5,10,20,40,60,80$ $\%$ of peak flux density value at $6.86 \mathrm{mJy}$.

best fit model gives the stellar velocity dispersion value $\sigma=189.9 \mathrm{~km} \mathrm{~s}^{-1}$.

\section{RESULTS}

\section{$5.1 \quad$ Radio Images}

The radio images at $6,8.5,11.5$ and $15 \mathrm{GHz}$ are shown in Figures 2, 3, 4, 5 and 6 The uniform weighted images gave the optimum spatial resolution and flux densities but we used naturally weighted map at $8.5 \mathrm{GHz}$ to examine the Sshaped structure (Figure 4). The 6 and $15 \mathrm{GHz}$ radio images have two distinct radio lobes and hotspots on either side of the nucleus (Figure 4 and 6). The hotspot south of the nucleus has a larger flux density of $11.9 \mathrm{mJy}$ (see Table 3 ) suggesting that it is the closer jet and is curved towards the east. The increased brightness could be indicative of doppler boosting. The $8.5 \mathrm{GHz}$ (Figure 3 ) and $11.5 \mathrm{GHz}$ (Figure 5 ) images reveal a core lying between the radio lobes. The corejet structure is completely resolved at these frequencies and the deconvolved core has a size of $\sim 0.1^{\prime \prime}$ or 116 pc (Table 3). However, at $8.5 \mathrm{GHz}$ the naturally weighted image (Figure 4) shows the full extent of the helical S-shaped jet structure out to a radius of approximately $\sim 1.5^{\prime \prime}$ or $1.74 \mathrm{kpc}$. Interestingly, both the high and low resolution maps show distinctive curved radio jets, thus producing the S-shaped morphology for the radio source. The helical structure of the jets is very clear since the hotspots lie along the NW$\mathrm{SE}$ direction, but the extended radio emission lies along the NE-SW direction.

\section{$5.22 \mathrm{D}$ decomposition of galaxy image}

Dual AGN are rare in spirals, and S-shaped radio sources are usually associated with major mergers of elliptical galaxies (e.g. NGC 326). Hence, it is important to see if 2MASXJ1203

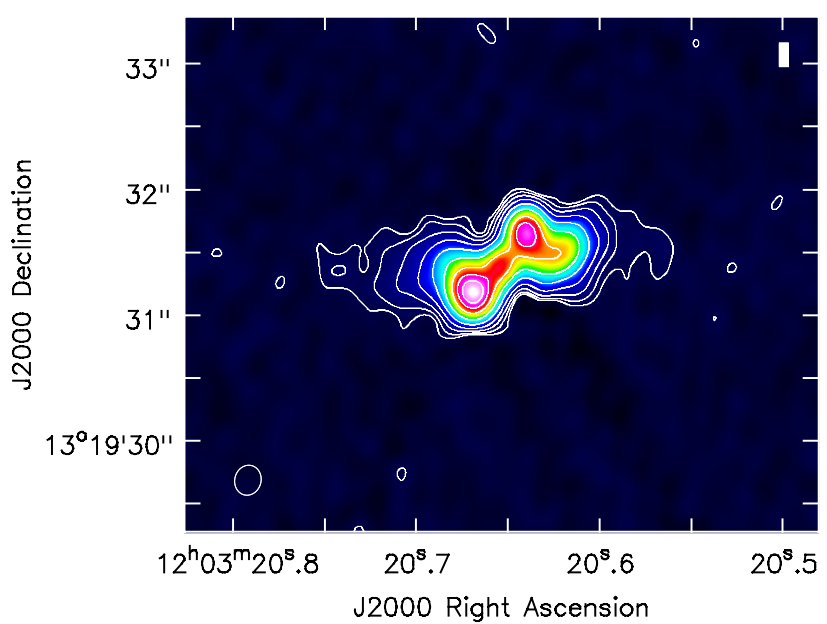

Figure 4. The naturally weighted $8.5 \mathrm{GHz}$ EVLA image of 2MASXJ12032061+131931. The beam size is $0.24^{\prime \prime} \times 0.21^{\prime \prime}$. The contour levels correspond to $0.60,1.25,2.5,5,10,20,40,60,80 \%$ of peak flux density at $8.20 \mathrm{mJy}$.

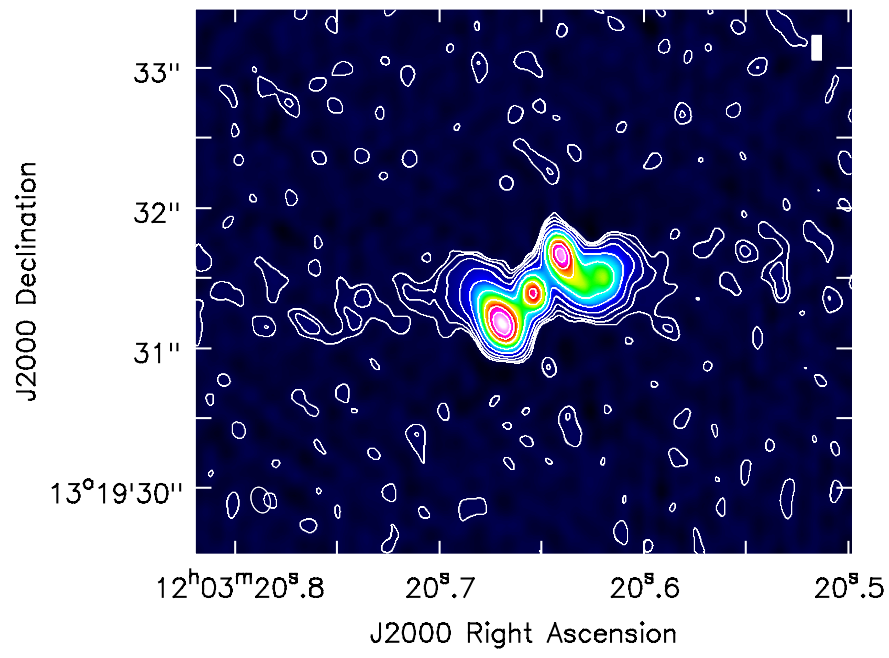

Figure 5. The uniform weighted radio contour map 2MASXJ12032061+131931 at $11.5 \mathrm{GHz}$. The beam size is $0.19^{\prime \prime} \times 0.13^{\prime \prime}$. The contour levels correspond to 0.60, 1.25, 2.5, $5,10,20,40,60,80 \%$ of peak flux density of value $4.90 \mathrm{mJy}$.

is an elliptical or spiral galaxy. To determine the morphological class of 2MASXJ1203, we used GALFIT to first fit a single Sersic profile (which is used for elliptical galaxies) to the SDSS I band image. The fit is shown in Figure 7 The fit appears good and has a $\chi^{2}$ value of 1.097 . However according to Fathi et al. (2010) 2MASXJ1203 could be an S0 type galaxy. Therefore, we tried with two components simultaneously - a Sersic profile for a bulge and an exponential profile for a disk. While the two component fit results in an 


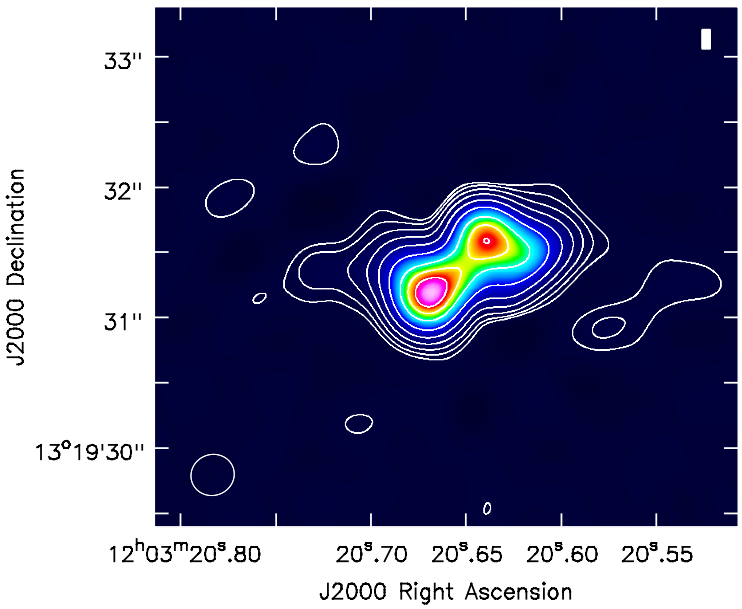

Figure 6. The naturally weighted radio contour map 2MASXJ12032061+131931 at $15 \mathrm{GHz}$. The beam size is $0.33^{\prime \prime} \times$ $0.31^{\prime \prime}$. The contour levels correspond to $0.60,1.25,2.5,5,10,20$, $40,60,80 \%$ of peak flux density value of $7.30 \mathrm{mJy}$.

identical $\chi^{2}$ value, the residual image looks better compared to the single component fit. GALFIT therefore favours the disky morphology for the host galaxy. However, the exposure time for the SDSS image is very short. A deeper image is required to confirm the morphological class of 2MASXJ1203.

\subsection{SMBH mass, Eddington ratio and Star Formation Rate}

We have used the velocity dispersion of the underlying stellar component to calculate the total mass of the central bulge which is also an upper limit to the mass of the dual SMBHs. Using the nuclear stellar velocity dispersion $\sigma$ that we derived (Section 4) from the SDSS DR12 spectrum (Figure 1) and the M- $\sigma_{\star}$ relation in McConnell \& Ma (2013), we obtained an upper limit of $M_{B H}=(1.56 \pm 0.26) \times 10^{8} M_{\odot}$ for the SMBHs. Furthermore, we used an approximate model of a binary system with the components $M_{A}$ and $M_{B}$ in circular orbits about a common center of mass to obtain a very rough approximation for the individual $\mathrm{SMBH}$ masses. There are several assumptions in this calculation - (i) we have assumed that there has been a major merger and the bulges have relaxed and hence the $\mathrm{M}-\sigma$ relation is valid, (ii) the BHs are rotating in a Keplerian disk with a separation of $0.1-2 \mathrm{kpc}$ and the [O III] peaks trace their relative velocity, (iii) both SMBHs are accreting at a similar Eddington rate. According to Kepler's law, for a binary system the orbital velocity ratio of the two components is given by Wang et al. (2009)

$V_{A} / V_{B}=M_{B} / M_{A}=\Delta \lambda_{A} / \Delta \lambda_{B}=2.57 / 2.37=1.084$.

Here $V_{A}$ and $V_{B}$ are the Doppler redshift and blueshift velocities of the [O III] line. $\Delta \lambda_{A}$ and $\Delta \lambda_{B}$ are the Doppler redshift and blueshift of the [O III] line in units of $\AA$ which have values of 2.57 and 2.37 respectively (Wang et al. 2009). Using the total mass to be $(1.56 \pm 0.26) \times 10^{8} M_{\odot}$ the individual SMBHs are $\sim 0.82 \times 10^{8} M_{\odot}$ and $\sim 0.74 \times 10^{8} M_{\odot} . \mathrm{In}$ general, however, a constant separation between the SMBHs will not perturb the accretion disks and produce AGN ac- tivity; also the orbits are more likely to be elliptical than circular. We note that this calculation only gives a rough estimate of the individual SMBH masses since there is no clear signature of a major merger in the optical image, as mentioned in Section 2 The separation is also not confirmed as we will discuss later in Section 6.2

We have obtained the blueshifted $\left(16.6 \times 10^{-16}\right.$ erg $\left.\mathrm{cm}^{-2} \mathrm{~s}^{-1}\right)$ and redshifted $\left(19.1 \times 10^{-16} \mathrm{erg} \mathrm{cm}^{-2} \mathrm{~s}^{-1}\right)$ narrow-line [O III] $\lambda 5008$ fluxes from Ge et al. (2012). The total flux density is $35.7 \times 10^{-16} \mathrm{erg} \mathrm{cm}^{-2} \mathrm{~s}^{-1}$. The [O III] luminosity turns out to be $2.79 \times 10^{40} \mathrm{erg} \mathrm{s}^{-1}$. We have estimated the bolometric luminosity $\left(L_{b o l}\right)$ using the relation from Heckman et al. (2004): $L_{b o l} / L_{\left[O_{\text {III }}\right]} \approx 3500$ and it's value is $L_{b o l}=9.79 \times 10^{43} \mathrm{erg} \mathrm{s}^{-1}$. For a BH mass $(1.56 \pm 0.26) \times 10^{8} M_{\odot}$, the Eddington luminosity $\left(\equiv 1.25 \times 10^{38} M_{B H} / M_{\odot}\right)$ is $\approx 1.87 \times 10^{46} \mathrm{erg} \mathrm{s}^{-1}$. The Eddington ratio in 2MASXJ1203 $\left(L_{b o l} / L_{E d d}\right)$ is $\sim 0.0052$ which is typical for Seyfert galaxies (e.g., Ho 2008).

We have derived the star formation rate (SFR) for the nuclear region in 2MASXJ1203 using the UV flux and $H_{\alpha}$ line emission. We have assumed a Salpeter initial mass function (IMF) (Salpeter 1955) and stellar mass limits of 0.1 to $100 M_{\odot}$ (Kennicutt 1998). The UV SFR is $\sim 0.105 M_{\odot} \mathrm{yr}^{-1}$ for the GALEX NUV flux of our target which is $9.60 \mu \mathrm{Jy}$. We have obtained the $H_{\alpha}$ flux from SDSS nuclear spectrum of 2MASXJ1203 and it has a value of $25.3 \times 10^{-16} \mathrm{erg} \mathrm{cm}^{-2} \mathrm{~s}^{-1}$. This yields a nuclear SFR of $\sim 0.156 M_{\odot} \mathrm{yr}^{-1}$. The full $H_{\alpha}$ luminosity is from both star formation as well as from AGN activity. In order to obtain the SFR, we need to subtract the AGN contribution from the $H_{\alpha}$ line luminosity. The contribution from AGN activity depends on selection criteria (Fujita et al. 2003). We have used Tresse et al. (1996) to get the AGN contribution in low redshift galaxies which is $8 \%$ to $17 \%$. We have used these limits to subtract the AGN contribution and calculate the SFR using only stellar emission. The corrected SFR range is $0.130-0.138 \mathrm{M}_{\odot} \mathrm{yr}^{-1}$. The GALEX UV flux is from the entire galaxy and the SDSS $\mathrm{H}_{\alpha}$ emission is from the central region $\left(\sim 3^{\prime \prime}\right.$ diameter SDSS fibre). The fact that both the UV flux and $H_{\alpha}$ line fluxes give similar SFRs indicates that the star formation is confined to the central region of the galaxy. Similar SFRs have been derived from infrared data in other Seyfert galaxies (e.g., Kharb et al. 2016).

We also checked the optical variability of 2MASXJ1203 using the Catalina Real-time Transient Survey (CRTS $)^{7}$ (Drake et al.2009). CRTS is a large sky, optical survey which publishes the light curves of sources within minutes of their observations. Thus the flux variation of transient sources with time can be obtained from CRTS data. However, for 2MASXJ1203 no clear periodicity is evident from its CRTS data.

\subsection{Spectral index}

The core has a spectral index value of $\alpha_{8.5}^{11.5} \sim-0.60 \pm$ 0.02 between $8.5 \mathrm{GHz}$ and $11.5 \mathrm{GHz}$ which is moderately steep. Steep spectrum cores have sometimes been observed in Seyfert galaxies (Peck \& Taylor 2001). The SE hotspot has a spectral index of $\alpha_{8.5}^{11.5} \sim-0.76 \pm 0.01$ and the NW

7 http://nesssi.cacr.caltech.edu/DataRelease/ 

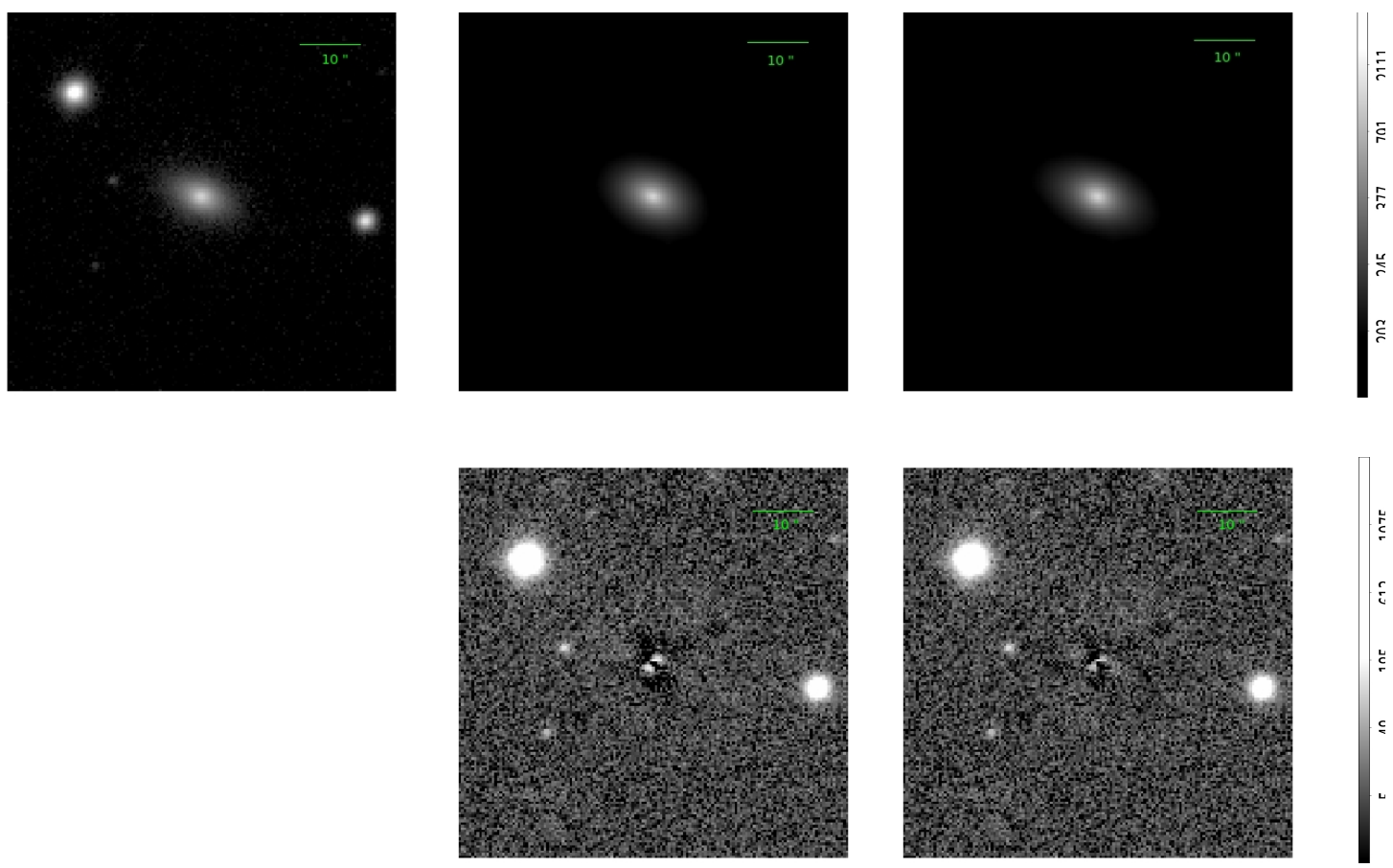

Figure 7. The optical image of the host galaxy of 2MASXJ1203 is fitted with Sersic and exponential profiles. Starting from the top left panel and moving from left to right, is the original galaxy image, the model with a single Sersic profile, and the model with a Sersic plus an exponential profile. The second row presents the residual images from the models presented immediately above. The color bars at the extreme right apply to all images in the respective rows. In all images, North is to the top and East towards the left.

one $\alpha_{8.5}^{11.5} \sim-0.72 \pm 0.02$ respectively (Figure 8). We have obtained the spectral index values of jets using the $6 \mathrm{GHz}$ and $15 \mathrm{GHz}$ images. The values are $\alpha_{6}^{15} \sim-0.68 \pm 0.01$ and $\alpha_{6}^{15} \sim-0.61 \pm 0.01$ for the SE and NW hotspot respectively (Figure 9). These spectral index values for the lobes are typical of lobes associated with jets in large radio galaxies (e.g., Laing \& Peacock 1980, Dennett-Thorpe et al. 1999. Kharb et al. 2008).

\subsection{Modeling the helical Jet-precession}

S- or Z- shaped jets have been observed in many galaxies. Hutchings, Price \& Gower (1988) found that almost $30 \%$ of all quasars with $\mathrm{z}<1$ and radio structures show an Sshape. Pronounced S-shaped jets are often termed helical jets and can be explained in terms of precessing jets (Ekers et al. 1978, Parma, Ekers \& Fanti 1985). We have modeled the helical jets in 2MASXJ1203 using the Hjellming \& Johnston (1981) model, since it is simple and does not assume any origin for the jet precession. The model was used for SS433 but it can be used for extra-galactic jets as well. From visual inspection we have derived the proper motion plot that matches the radio image in the following way. We started with a typical jet advance speed of $0.03 \mathrm{c}$ (Ulvestad 2003) and varied the inclination angle $i$ and half opening angle $(\psi)$ from 0 to $90^{\circ}$. The best fitting model values are $i=52^{\circ}, \psi=21^{\circ}, v=0.023 \mathrm{c}$, precession period $P=0.95 \times 10^{5}$ years. Such precession timescales have been found for the jets of the Seyfert galaxy Mrk6 as well (Kharb et al. 2006). The angle of rotation required to rotate the geometrical model to align it with the $11.5 \mathrm{GHz}$ image is $\chi=33^{\circ}$. The proper motion is plotted for a jet precession timescale of $t=1.5 \times 10^{5}$ years in Figure 10 . To estimate the errors associated with our precession model we determined the range of parameters that can fit the radio morphology in the following way. We fixed all the parameters except one and then varied this parameter until the visually identified fit got significantly worse (Steenbrugge \& Blundell 2008). The parameter ranges that we obtained were $i=52^{\circ} \pm 5^{\circ}$, $\psi=21^{\circ} \pm 2^{\circ}$ and $\chi=33^{\circ} \pm 3^{\circ}$. The range of precession period is $P=(0.95 \pm 0.05) \times 10^{5}$ years. The best fitting velocity which gives jets of the same size as in the radio image with the above parameters is $v=0.023 c$. Small changes in jet velocity around this value do not change the shape much. But if $v$ is continuously increased, the jets start showing relativistic effects which make the jet structure look increasingly asymmetric.

One of the most common explanations for jet precession is the presence of a binary black hole system, where the interaction of the black holes perturbs the spin of one or both black holes resulting in precessing jets (Blundell \& Bowler 2004 Romero et al. 2000 Roos, Kaastra \& Hummel 1993). Apart from binary black-hole systems, helical jets can arise when an accretion disk is irradiated by the central AGN and becomes unstable and warped thus producing jet precession (Pringle 1996, Livio \& Pringle 1997); it can also be due to the net change in the spin of the SBMHs which affects the orientation of the radio jets Rees 1978 Natarajan \& Pringle 1998). Models with accretion disks of single AGN have also been developed ( $\mathrm{Lu} 1990)$. Black-hole mergers can 


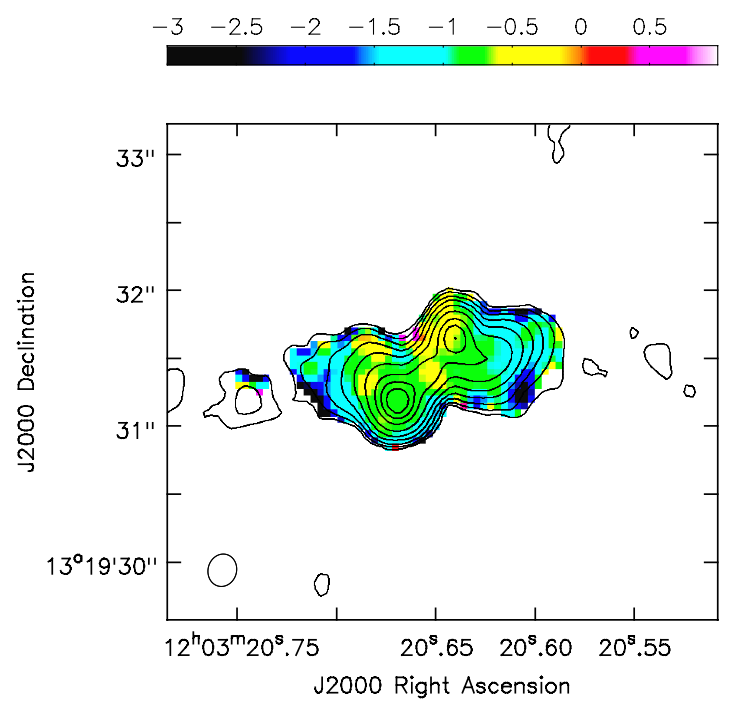

Figure 8. The 8.5-11.5 GHz spectral index image in colour, superimposed by $8.5 \mathrm{GHz}$ radio contours with levels corresponding to $60,1.25,2.5,5,10,20,40,60,80 \%$ of peak flux density value at $8.2 \mathrm{mJy}$.

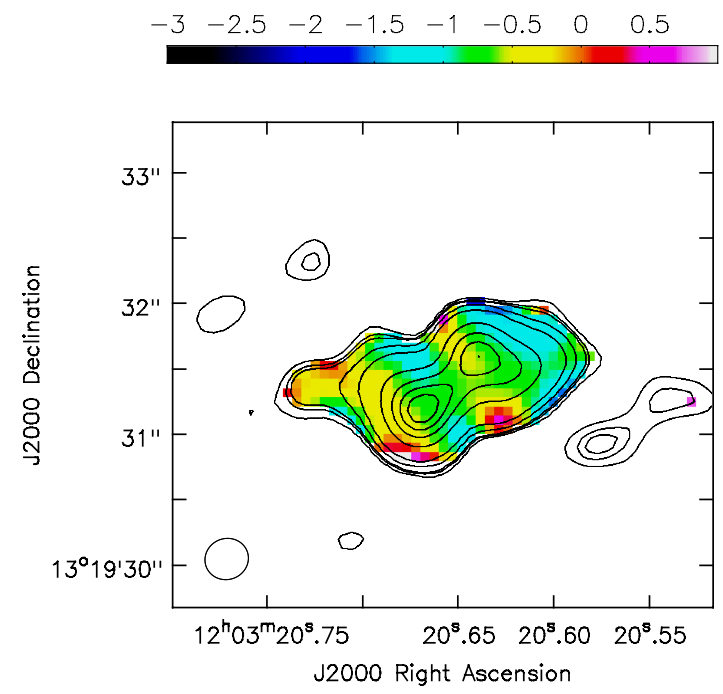

Figure 9. The 6-15 GHz spectral index image in colour, superimposed by $15 \mathrm{GHz}$ radio contours with levels corresponding to $60,1.25,2.5,5,10,20,40,60,80 \%$ of peak flux density value at $7.3 \mathrm{mJy}$.

result in short-timescale redirection of the jet axis (Merritt \& Milosavljević 2005) and this may also lead to bent radio jets, but these are X-shaped rather than S-shaped jets.

\subsection{Equipartition Estimates and Lifetimes}

We have obtained the magnetic field corresponding to the minimum total energy of the synchrotron emitting plasma which is close to the equipartition of the energy in particles and the magnetic field (Burbidge 1959). Using the radio luminosity (O'Dea \& Owen 1987), we obtain the following expression.

$L_{\text {rad }}=1.2 \times 10^{27} D_{L}^{2} S_{0} \nu_{0}^{-\alpha}(1+z)^{-(1+\alpha)}\left(\nu_{u}^{1+\alpha}-\nu_{l}^{1+\alpha}\right)(1+\alpha)^{-1}$

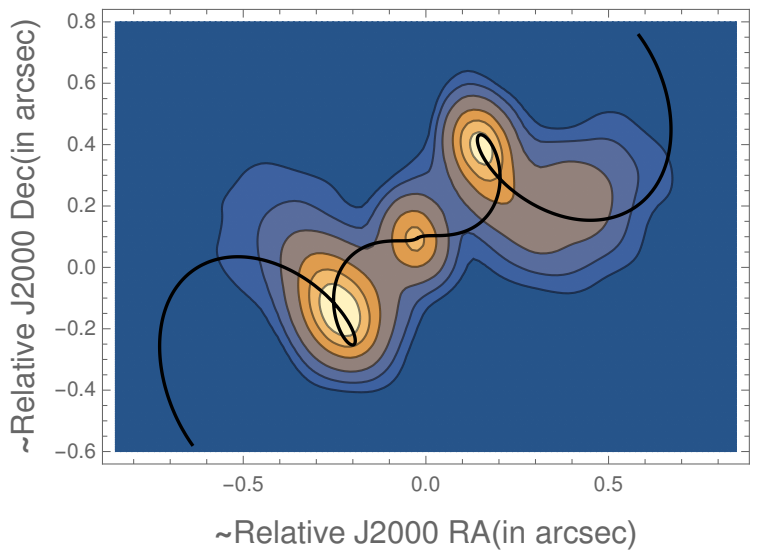

Figure 10. The uniform weighted $11.5 \mathrm{GHz}$ radio image of 2MASXJ12032061+131931 in color, superimposed by the precessing jet model of Hjellming \& Johnston (1981) in black. The bestfit parameters are described in Section 5.5

$B_{\text {min }}=\left[2 \pi(1+k) c_{12} L_{\text {rad }}(V \phi)^{-1}\right]^{2 / 7}$

Where $\mathrm{L}_{\text {rad }}$ is the radio luminosity in erg $\mathrm{s}^{-1}, \mathrm{D}_{L}$ is the luminosity distance in Mpc, $\mathrm{z}$ is the redshift (Table 1), $\mathrm{S}_{0}$ is the total flux density in $\mathrm{Jy}, \nu_{0}$ is the frequency in $\mathrm{Hz}$, $\nu_{u}$ and $\nu_{l}$ are the upper and lower cutoff frequencies respectively in $\mathrm{Hz}, \mathrm{k}$ is the ratio of the relativistic proton to relativistic electron energy, $\mathrm{V}$ is the source volume, $\mathrm{c}_{12}$ is a constant depending on the spectral index and frequency cutoffs (Pacholczyk 1970), $\phi$ is the volume filling factor and $\mathrm{B}_{\min }$ is the magnetic field at minimum pressure in Gauss. We have used the total flux density $\mathrm{S}_{0}=0.29 \mathrm{Jy}$ from the $11.5 \mathrm{GHz}\left(\nu_{0}\right)$ image (Figure 5). The minimum magnetic field is calculated using the following assumptions: the radio spectrum extends from $100 \mathrm{MHz}$ to $15 \mathrm{GHz}$ with a spectral index value of $\alpha=-1$ (Figure 8). The relativistic electron and proton have similar energy i.e $\mathrm{k}=1$. The jets are uniformly filled with relativistic particle and magnetic field i.e $\phi=1$. We have assumed cylindrical symmetry for the jet and calculated the volume, $V=\pi(w / 2)^{2} l$, using a length of $l \sim 3 \mathrm{kpc}$ and a width of $w \sim 0.4 \mathrm{kpc}$. The parameter $c_{12}$ is $6.5 \times 10^{7}$ for our observed frequency. The estimated total radio luminosity is $1.9 \times 10^{41} \mathrm{erg} \mathrm{s}^{-1}$. We obtain a minimum magnetic field of $105 \mu \mathrm{G}$ for a plasma filling factor of $\phi=1$.

We have used the relation from van der Laan \& Perola (1969) to estimate the lifetime of electrons in the radio component undergoing both synchrotron radiative and inverse-Compton losses due to cosmic microwave background (CMB) photons,

$$
t \approx \frac{2.6 \times 10^{4} B_{\min }{ }^{1 / 2}}{\left(B_{\min }{ }^{2}+B_{R}^{2}\right)[(1+z) \nu]^{1 / 2}}
$$

where $\mathrm{B}_{\min }$ is the minimum magnetic field from equipartition in $\mathrm{G} ; \mathrm{B}_{R} \simeq 4 \times 10^{-6}(1+z) \mathrm{G}$ is the magnetic field equivalent to the radiation, which was assumed to be predominantly CMB photons and $\nu$ is the electron radiation frequency in $\mathrm{Hz}$. For the estimated magnetic field of $105 \mu \mathrm{G}$ at $11.5 \mathrm{GHz}$ we obtain the lifetime of electrons to be $\mathrm{t} \approx 2.1 \times 10^{5}$ yrs.

The age of the source can also be estimated via the 
spectral ageing analysis (Myers \& Spangler 1985). We have used Figure 3 from Myers \& Spangler (1985) to obtain a rough estimate of $\mathrm{X}_{0}$ which is the measure of maturity of synchrotron losses. We have used the $\alpha_{6}^{15}$ values (Figure 9 at the initial hotspots and where jet falls below $3 \sigma$; these frequencies match those used in Figure 3 of Myers \& Spangler (1985). We have used the Kardashav-Poacholczyk (KP) model where assumes an isotropic pitch angle distribution. The initial electron energy is from synchrotron radiation which follows a power law. The magnetic field is assumed to be the equipartition value. The estimated timescale is $\mathrm{t}>1.3 \times 10^{5} \mathrm{yrs}$. This is a lower limit since only the hotspot and the tail were considered instead of the entire core-jet structure. We have calculated the jet speed using the synchrotron time i.e $2 \times 10^{5}$ yrs and the size of one-side jet i.e $1.5 \mathrm{kpc}$ which comes out to be $\beta=0.023$. The calculated jet advance speed is the same as was obtained from the precession model in Section 5.5. The timescale of the order of $\sim 10^{5}$ is also consistent with the precession timescale obtained in Section 5.5 .

\section{DISCUSSION}

Our radio observations show that 2MASXJ1203 has a corejet structure with S-shaped or helical jets. In this section we discuss the mechanisms that could explain the radio observations of the DPAGN in 2MASXJ1203.

\subsection{Mechanisms Causing S-shaped Core Jet Structures}

In the literature, $\mathrm{Z}$ or $\mathrm{S}$-shaped radio galaxies are referred to as X-shaped galaxies (Cheung \& Springmann 2007). However, it is very difficult to explain this morphology with the models for X-shaped galaxies which consist mainly of (i) spin flip of a SMBH (Natarajan \& Pringle 1998) or (ii) backflow of plasma in radio jets (Leahy \& Williams 1984). In the back-flow model, the ISM of the host elliptical galaxy exerts a buoyancy pressure on the back-flowing plasma causing secondary wings of plasma to form from the terminal shocks evolving in the hot medium (Capetti et al. 2002). The rapid cocoon expansion along the direction of the hosts minor axis produces the $\mathrm{X}$-shaped morphology. The relationship between the optical axis and wing orientation indicates that the formation of the XRSs is intimately related to the host galaxy's geometry (Gillone, Capetti \& Rossi 2016). These results strengthen the interpretation that the X-shaped morphology in radio-sources has a hydrodynamical origin. The back-flow model can explain the radio structures associated with the large FR I Fanaroff \& Riley (1974) galaxies. But the model is not able to explain why the secondary lobes are larger than the primary lobes in many radio galaxies such as NGC 326, 4C+00.58 (Hodges-Kluck et al. 2010). However, 2MASXJ1203 cannot be classified as an FRI radio source due to its small size and the secondary wings are larger than the primary. There is also no evidence of cocoon formation in the radio images. Therefore, the back-flow model cannot explain the radio morphology of 2MASXJ1203. GopalKrishna, Biermann \& Wiita (2003) have proposed a different model which can explain both X and Z-shaped sources. In this model, as a galaxy spirals into the nucleus of the host galaxy, it perturbs the axisymmetric disk potential, causing disk rotation in the interstellar medium (ISM) of the host galaxy (Noel-Storr et al. 2003). The gas in the disk will interact with the jets causing them to bend at large radii this may appear as jet precession i.e. $\mathrm{Z}$ or $\mathrm{X}$ shaped radio jets. A good example is the Z-shaped galaxy NGC 3801 (Das et al. 2005). Hota et al. (2009) showed that Z-shaped radio jets in NGC 3801 have gone through a recent merger and it has a large fast rotating gas disk interacting with jets. In our case, however, there is no indication of jet-ISM interaction and our radio jets have small radii of $\sim 1.5 \mathrm{kpc}$. Thus, this model is probably not the explanation for the S-shaped radio jet morphology in 2MASXJ1203.

A helical jet structure can also be due to jet precession during the lifetime of a radio source (Ekers et al. 1978). The jet precession model fits the radio image of 2MASXJ1203 (see Section 5.5) and the calculated jet precession timescale is similar to the age of the electrons in the lobes i.e $10^{5}$ yrs (see Section 5.6). Therefore, jet precession is the most probable explanation for the S-shaped radio structure of 2MASXJ1203. We discuss jet precession in greater detail in the next section.

\subsection{Mechanisms Causing Jet Precession}

S-symmetry due to precession has long been predicted associated with the presence of binary SMBHs (Begelman, Blandford \& Rees 1980). The secondary BH can induce the rapid precession in the inner region of the primary accretion disk (Romero et al. 2000). The motion of the secondary $\mathrm{BH}$ around the primary $\mathrm{BH}$ can also cause the orbit to precess resulting in helical or S-shaped radio jets (Roos, Kaastra \& Hummel 1993, Valtonen et al. 2016). A good example is the BL Lac object Mrk 501 that has helical jets which can be explained by binary SMBH models (Villata \& Raiteri 1999). We have calculated the separation of the binary system which can give the precession period of $10^{5}$ years. The relation, $P_{\text {prec }} \sim 600 r_{16}{ }^{5 / 2}(M / m) M_{8}{ }^{-3 / 2}$ yr Begelman, Blandford \& Rees 1980) gives a separation of $\sim 0.02 \mathrm{pc}$, where $r_{16}$ is the separation in units of $10^{16} \mathrm{~cm}, M_{8}$ is the mass of the primary SMBH in units of $10^{8} M_{\odot}$ and $(\mathrm{M} / \mathrm{m})$ is the mass ratio of primary $\mathrm{SMBH}$ to secondary SMBH. We have used the mass ratio 1.084 and $M_{8} \sim 0.82$ (Section 5.3). Therefore, there could be a second SMBH at a separation of 0.02 pc in 2MASXJ1203, which we cannot detect with the present day telescopes. The other possibility could be that there exists a dual SMBH system and the secondary SMBH has passed the primary $\mathrm{SMBH}$ in the past; this could have induced the precession in the jet of the primary SMBH. Here the secondary SMBH may not have sufficient radio flux density to be detected in our observation; higher resolution observations however, could detect it, if present. In the case of 2MASXJ1203, there appears to be a second NIR Core in Keck NIRC2 image (McGurk et al. 2015). The image contains a second core at a separation of $2.1 \mathrm{kpc}$ from the nucleus. However, spectroscopic observations have not been carried out. The second faint core could form a DAGN system with the nuclear SMBH in the bulge. However, we cannot rule out an AGN-starburst system either. If the faint NIR core is a SMBH it does not have enough radio flux density to be detected in our maps. This second SMBH could cause the jet precession in 2MASXJ1203. 
Though binary models are interesting, a single AGN model can also explain the helical jet morphology. On the basis of the Sarazin, Begelman \& Hatchett (1980) model for SS433, Lu (1990) suggested that a tilted accretion disk can also produce jet precession. This model of a tilted accretion disk around a single AGN gives a relation between the precession period and the luminosity. The radio source 1946+708 has jets with a tilted disk (Peck \& Taylor 2001) and the observed morphology follows the period-luminosity relation of $\mathrm{Lu}(1990)$. The precession period is usually calculated using several precession models which have very large uncertainties and the magnitude values also differ from literature to literature. We have used the period-luminosity relation where the absolute B-band magnitude of our target ${ }^{8}$ is $M_{a b s}=-20.34$ and the calculated precession period is $\sim 10^{5}-10^{9}$ yrs. We note that the timescale uncertainties are very large. However, our jet precession model time estimate of $\sim 10^{5}$ yrs that was obtained in Section 5.5 falls within this time range. It is possible that the accretion disk in 2MASXJ1203 has become warped or tilted due to nonuniform irradiation from the AGN. Such radiation can cause the accretion disk to become unstable and warped, resulting in jet precession (Pringle 1997). In radio images we have not detected the second core so we cannot distinguish between these two mechanisms of precession. Thus in summary the jet precession in 2MASXJ1203 can be due to a close SMBH binary at a separation of $0.02 \mathrm{pc}$, a dual system in which a close passing of an SMBH has induced the precession or a single AGN with an warped accretion disk that has given rise to jet precession.

\subsection{Is 2MASXJ1203 a CSS/CSO source?}

The other class of radio sources into which 2MASXJ1203 falls is that of the compact steep spectrum (CSS) objects or compact symmetric objects (CSO). Gigahertz peakedspectrum $(\mathrm{GPS})(\leqslant 1 \mathrm{kpc})$ and CSS $(\leqslant 20 \mathrm{kpc})$ sources are small bright sources with steep spectra and are young $\left(\leqslant 10^{5}\right.$ yrs). CSS and GPS sources can probe the NLR of the host galaxy (O'Dea 1998). CSO are radio objects which have compact symmetric double lobes that extend to small galactic radii of $\leqslant 1 \mathrm{kpc}$ (Wilkinson et al. 1994). CSO are a subclass of GPS with symmetric jets. CSS are also symmetric in structure. Fanti et al. (1995); Readhead et al. (1996) have proposed that GPS and CSS are the evolutionary stages of large radio galaxies i.e GPS $\rightarrow$ CSS $\rightarrow$ large radio galaxies. Some CSOs show S-symmetry (Deane et al. 2014) which can be signature of binary SMBHs (Begelman, Blandford \& Rees 1980). The binary AGN with the smallest separation, 0402+379, is a CSO (Rodriguez et al. 2006). GopalKrishna (1995) have suggested that a merger, either with a dwarf galaxy or a secondary $\mathrm{SMBH}$, could disturb the torus funnel in the AGN and give rise to jet misalignment. Such a distorted torus can give rise to a GPS source. We have calculated the break frequency of 2MASXJ1203 using $t_{s}=1610 B^{-3 / 2} \nu_{b}^{-1 / 2} \mathrm{Myr}$ from (Carilli et al. 1991), where $\mathrm{t}_{s}$ is the synchrotron age , $\mathrm{B}$ is the magnetic field and $\nu_{B}$ is the break frequency. We have used $\mathrm{t}_{s}=10^{5} \mathrm{yrs}$, $\mathrm{B}=105 \mu \mathrm{G}$ from Section 5.6. The calculated break frequency

8 http://leda.univ-lyon1.fr/ is $\nu_{B} \sim 0.55 \mathrm{GHz}$. This $\nu_{B}$ is in the break frequency range observed in CSO/CSS sources (Murgia 2003). The radio observations of 2MASXJ1203 show an S-shaped jet of total extent $\sim 3 \mathrm{kpc}$, the average spectral index $\alpha_{8.5}^{11.5} \sim-1, \nu_{B}$ is $0.55 \mathrm{GHz}$ and the estimated age is $\sim 10^{5} \mathrm{yrs}$. Thus 2MASXJ1203 is a CSS/CSO source and its S-symmetry could be consistent with the binary/dual SMBH scenario.

\subsection{Origin of Double-peaked [O III] line}

Double peaked emission lines from the NLR have been detected in nuclear galaxy spectra since the 1970s (Sargent 1972 Heckman et al. 1981) and are important for studies of NLR kinematics. It was previously thought that DPAGN emission lines are indicators of bipolar outflows or rotating disks (Greene \& Ho 2005). However, they are now thought to also indicate the presence of binary or dual AGN (Zhou et al. 2004 ) at the separations of $\sim 100$ pc to $\sim 10 \mathrm{kpc}$ respectively (Wang et al. 2009). Since the data of large spectroscopic surveys such as SDSS became available (Smith et al. 2010. Fu et al. 2012), there has been a greater interest in finding large sample of DPAGNs since they may represent DAGN. However, there are several other mechanisms that can produce double-peaked emission lines from a single AGN (Xu \& Komossa 2009). For example, jet-cloud interaction can also produce a DPAGN (Rosario et al. 2010). Smith et al. (2010) suggest that if two Gaussian components of a double-peaked [O III] line are symmetric than it can be explained by a rotating ring. Thus, it is very difficult to confirm the presence of dual/binary AGN just from DPAGN emission and one has to carry out high resolution imaging to confirm it. Tingay \& Wayth (2011) conducted a VLBI study of DPAGN to search for dual/binary AGN but they have not detected any. Comerford et al. (2012) used an additional criteria to detect DAGN from a sample of DPAGN; they used longslit spectroscopy to separate the outflow and rotating disks from candidate dual/binary AGN sources. They found 17 promising dual AGN candidates from 81 DPAGN sources. Müller-Sánchez et al. (2015) have tried to constrain the origin of DPAGN using VLA observations along with long-slit spectroscopic studies. They find that $15 \%$ of their sample are dual AGN and in $75 \%$ the DPAGN are due to gas kinematics. From these recent studies, it appears that DPAGN may not be promising candidates for dual/binary AGN.

In our study, we do not have a two dimensional [O III] map of the gas kinematics or high resolution long slit observations for our target. Therefore, we cannot be sure about the origin of the double-peaked emission lines in 2MASXJ1203 just from the SDSS spectrum. However, just to check if the double peaked [O III] lines could be due to two SMBHs, we used the velocity separation $\Delta v=292 \mathrm{~km} \mathrm{~s}^{-1}$ of the [O III] emission lines (Ge et al. 2012) to make an approximate estimate of the SMBH separation using $\Delta R \sim G M /(\Delta v)^{2}$ and used it to derive a precession timescale. The calculated separation for $M=10^{8} M_{\odot}$ is $\sim 8$ pc. Using this value in equation 7 from Begelman, Blandford \& Rees (1980) gives a precession period of $4.6 \times 10^{11}$ yrs which is more than the Hubble time. This value contradicts the model precession timescale (which is due to a binary of separation of $0.02 \mathrm{pc}$ ) as well as the synchrotron lifetimes of electrons in the radio jets, both of which are of the order of $\sim 10^{5} \mathrm{yr}$. Thus in the precession model it is not 
possible that a close binary is the origin of the double peaked emission lines in the SDSS spectrum of 2MASXJ1203. However, it could be that there is a dual system in which the precession has been induced during a close pass; in that case we cannot rule out the possibility that the DPAGN is due to two AGN. Alternatively, there is only a single AGN in which case the jet-ISM interaction is the probable origin of the DPAGN emission line. The kpc radio jets may be responsible for the double-peaks through jet-medium interaction.

\subsection{Detecting the dual/binary AGN in 2MASXJ1203}

The S-shaped radio morphology of 2MASXJ1203 is due to slow precession of jets during its lifetime and it is a CSS/CSO source. We have considered three scenarios for jet precession: (i) binary AGN at the separation of $0.02 \mathrm{pc}$, (ii) a dual AGN system where a close pass of the secondary $\mathrm{SMBH}$ in the past has given rise to the jet precssion or (iii) a single AGN with a tilted accretion disk. If the first case is true, i.e., the separation is 18 microarcseconds in the sky, we cannot resolve the second AGN with current ground-based VLBI telescopes. With our present data we are not able to rule out any of these possibilities but future higher resolution observations may help us if the SMBHs are at separation lying between $40 \leqslant \mathrm{~d} \leqslant 100 \mathrm{pc}$. If the separation is more than $100 \mathrm{pc}$, the second AGN does not have sufficient radio flux density since it has not been detected in our observations.

\subsection{The relevance of DPAGN to dual/binary AGN studies}

Recent high resolution radio observations have shown that DPAGN are not usually dual/binary AGN (Shen et al. 2011. Fu et al. 2011b) as indicated by earlier studies and DAGN appear to be far more elusive than thought. However, they are very important for understanding the nuclear disk kinematics and good indicators of $\mathrm{kpc}$ scale radio jets or outflows (Müller-Sánchez et al. 2011, Kharb et al. 2015). The lack of parsec scale resolution and the absence of radio or X-ray emission in a significant fraction of AGN makes it harder to detect DAGN. In some cases the NIR images or radio morphologies may have indirect signatures of the SMBH pair such as lopsided disks or S-shaped jets as in 2MASXJ1203. These cases need to be followed with higher resolution observations. Thus multi-wavelength and high resolution observations are essential to detect dual/binary AGN in DPAGN galaxies.

\section{SUMMARY}

We summarise the conclusions of our study below:

1. We have carried out radio imaging of the double-peaked emission line Seyfert 2 galaxy 2MASXJ1203 using EVLA at $6,8.5,11.5$ and $15 \mathrm{GHz}$. The 6 and $15 \mathrm{GHz}$ images show two distinct radio hotspots on either side of the optical nucleus.

2. The 8.5 and $11.5 \mathrm{GHz}$ images obtained via archival data, reveal the full extent of the emission and we are able to resolve the core jet structure. The radio jets have an S-shaped helical structure extending out to a radius of $\sim 1.5^{\prime \prime}(1.74 \mathrm{kpc})$ on either side of a deconvolved core of size $\sim 0.1^{\prime \prime}$ (116 parsec).

3. We have modeled the helical-jet structure using the Hjellming \& Johnston (1981) model. The best-fit jet advance speed is $0.023 \mathrm{c}$ and precession timescale is $\sim 10^{5} \mathrm{yrs}$. The half opening angle is $\psi=21^{\circ} \pm 2^{\circ}$ and inclination angle for the radio jets is $i=52^{\circ} \pm 5^{\circ}$.

4. We have calculated the minimum magnetic field value of $105 \mu \mathrm{G}$ and the electron lifetime of $\sim 10^{5}$ years from the equipartition theorem. This timescale matches the time the precessing jet was "on" in this Seyfert galaxy, providing support to the precession model.

5. 2MASXJ1203 is compact steep spectrum/compact symmetric object. Such sources have been suggested to be binary AGN in the literature.

6. The presence of S-shaped precessing radio jets in 2MASXJ1203 can be due to binary/dual SMBH or a single tilted SMBH with accretion disk. Double-peaked emission lines also can be due to binary/dual AGN or NLR kinematics of a single AGN. While the binary/dual SMBH scenario is supported by several suggestions, we are unable to rule out other possibilities with the present data. Future high resolution multi-wavelength (radio, X-ray, optical) observations are required to get a clearer picture for the double-peaked AGN, 2MASXJ1203.

We thank the referee for insightful comments that improved the paper. We acknowledge IIA for providing the computational facilities. Rubinur K. wants to thank Dr. Avijeet Prasad for helpful discussions. The National Radio Astronomy Observatory is a facility of the National Science Foundation operated under cooperative agreement by Associated Universities, Inc. This research has made use of the NASA/IPAC Extragalactic Database (NED), which is operated by the Jet Propulsion Laboratory, California Institute of Technology, under contract with the National Aeronautics and Space Administration. Funding for the Sloan Digital Sky Survey IV has been provided by the Alfred P. Sloan Foundation, the U.S. Department of Energy Office of Science, and the Participating Institutions. SDSS- IV acknowledges support and resources from the Center for HighPerformance Computing at the University of Utah. The SDSS web site is www.sdss.org. We acknowledge the usage of the HyperLeda database (http://leda.univ-lyon1.fr)

\section{References}

Begelman M. C., Blandford R. D., Rees M. J., 1980, Nature, 287, 307

Bianchi S., Chiaberge M., Piconcelli E., Guainazzi M., Matt G., 2008, Mon. Not. Roy. Astron. Soc., 386, 105

Blundell K. M., Bowler M. G., 2004, Astrophys. J. Lett., 616, L159

Burbidge G. R., 1959, Astrophys. J., 129, 849

Burke-Spolaor S., 2015, ArXiv e-prints

Capetti A., Zamfir S., Rossi P., Bodo G., Zanni C., Massaglia S., 2002, Astron. Astrophys., 394, 39

Cappellari M., Emsellem E., 2004, Pub. Astron. Soc. Pac., 116,138 
Carilli C. L., Perley R. A., Dreher J. W., Leahy J. P., 1991, Astrophys. J., 383, 554

Cheung C. C., Springmann A., 2007, in Astronomical Society of the Pacific Conference Series, Vol. 373, The Central Engine of Active Galactic Nuclei, Ho L. C., Wang J.-W., eds., p. 259

Comerford J. M., Gerke B. F., Stern D., Cooper M. C., Weiner B. J., Newman J. A., Madsen K., Barrows R. S., 2012, Astrophys. J., 753, 42

Comerford J. M., Pooley D., Barrows R. S., Greene J. E., Zakamska N. L., Madejski G. M., Cooper M. C., 2015, Astrophys. J., 806, 219

Das M., Vogel S. N., Verdoes Kleijn G. A., O'Dea C. P., Baum S. A., 2005, Astrophys. J., 629, 757

Deane R. P. et al., 2014, Nature, 511, 57

Dennett-Thorpe J., Bridle A. H., Laing R. A., Scheuer P. A. G., 1999, Mon. Not. Roy. Astron. Soc., 304, 271

Drake A. J. et al., 2009, Astrophys. J., 696, 870

Ekers R. D., Fanti R., Lari C., Parma P., 1978, Nature, 276,588

Fanaroff B. L., Riley J. M., 1974, Mon. Not. Roy. Astron. Soc., 167, 31P

Fanti C., Fanti R., Dallacasa D., Schilizzi R. T., Spencer

R. E., Stanghellini C., 1995, Astron. Astrophys., 302, 317

Fathi K., Allen M., Boch T., Hatziminaoglou E., Peletier R. F., 2010, Mon. Not. Roy. Astron. Soc., 406, 1595

Fu H., Myers A. D., Djorgovski S. G., Yan L., 2011a, Astrophys. J., 733, 103

Fu H., Yan L., Myers A. D., Stockton A., Djorgovski S. G., Aldering G., Rich J. A., 2012, Astrophys. J., 745, 67

Fu H. et al., 2011b, Astrophys. J. Lett., 740, L44

Fujita S. S. et al., 2003, Astrophys. J. Lett., 586, L115

Ge J.-Q., Hu C., Wang J.-M., Bai J.-M., Zhang S., 2012, Astrophys. J. Lett., 201, 31

Gergely L. Á., Biermann P. L., 2009, Astrophys. J., 697, 1621

Gerhard O. E., 1993, Mon. Not. Roy. Astron. Soc., 265, 213

Gillone M., Capetti A., Rossi P., 2016, Astron. Astrophys., 587, A25

Gopal-Krishna, 1995, Proceedings of the National Academy of Science, 92, 11399

Gopal-Krishna, Biermann P. L., Wiita P. J., 2003, Astrophys. J. Lett., 594, L103

Graham M. J. et al., 2015, Nature, 518, 74

Greene J. E., Ho L. C., 2005, Astrophys. J., 627, 721

Heckman T. M., Kauffmann G., Brinchmann J., Charlot S., Tremonti C., White S. D. M., 2004, Astrophys. J., 613, 109

Heckman T. M., Miley G. K., van Breugel W. J. M., Butcher H. R., 1981, Astrophys. J., 247, 403

Hjellming R. M., Johnston K. J., 1981, Astrophys. J. Lett., 246, L141

Ho L. C., 2008, Annu. Rev. Astron. Astrophys., 46, 475

Hodges-Kluck E. J., Reynolds C. S., 2012, Astrophys. J., 746,167

Hodges-Kluck E. J., Reynolds C. S., Miller M. C., Cheung C. C., 2010, Astrophys. J. Lett., 717, L37

Hopkins P. F., Hernquist L., 2009, Astrophys. J., 694, 599 Hota A., Lim J., Ohyama Y., Saikia D. J., Dihn-v-Trung, Croston J. H., 2009, in Astronomical Society of the Pacific Conference Series, Vol. 407, The Low-Frequency Radio
Universe, Saikia D. J., Green D. A., Gupta Y., Venturi T., eds., p. 104

Hudson D. S., Reiprich T. H., Clarke T. E., Sarazin C. L., 2006, Astron. Astrophys., 453, 433

Hutchings J. B., Price R., Gower A. C., 1988, Astrophys. J., 329, 122

Junkkarinen V., Shields G. A., Beaver E. A., Burbidge E. M., Cohen R. D., Hamann F., Lyons R. W., 2001, Astrophys. J. Lett., 549, L155

Kennicutt, Jr. R. C., 1998, Annu. Rev. Astron. Astrophys., 36,189

Kharb P., Das M., Paragi Z., Subramanian S., Chitta L. P., 2015, Astrophys. J., 799, 161

Kharb P., O'Dea C. P., Baum S. A., Colbert E. J. M., Xu C., 2006, Astrophys. J., 652, 177

Kharb P., O'Dea C. P., Baum S. A., Daly R. A., Mory M. P., Donahue M., Guerra E. J., 2008, Astrophys. J. Lett., 174, 74

Kharb P., Srivastava S., Singh V., Gallimore J. F., IshwaraChandra C. H., Hota A., 2016, ArXiv e-prints

Komossa S., 2006, Astrophys. J. Lett., 77, 733

Komossa S., Burwitz V., Hasinger G., Predehl P., Kaastra J. S., Ikebe Y., 2003, Astrophys. J. Lett., 582, L15

Koss M., Mushotzky R., Treister E., Veilleux S., Vasudevan

R., Trippe M., 2012, Astrophys. J. Lett., 746, L22

Laing R. A., Peacock J. A., 1980, Mon. Not. Roy. Astron. Soc., 190, 903

Leahy J. P., Williams A. G., 1984, Mon. Not. Roy. Astron. Soc., 210, 929

Lehto H. J., Valtonen M. J., 1996, Astrophys. J., 460, 207

Liu T. et al., 2015, Astrophys. J. Lett., 803, L16

Liu X., Civano F., Shen Y., Green P., Greene J. E., Strauss

M. A., 2013, Astrophys. J., 762, 110

Liu X., Greene J. E., Shen Y., Strauss M. A., 2010, Astrophys. J. Lett., 715, L30

Livio M., Pringle J. E., 1997, Astrophys. J., 486, 835

Lu J. F., 1990, Astron. Astrophys., 229, 424

Makarov D., Prugniel P., Terekhova N., Courtois H., Vauglin I., 2014, Astron. Astrophys., 570, A13

McConnell N. J., Ma C.-P., 2013, Astrophys. J., 764, 184 McGurk R. C., Max C. E., Medling A. M., Shields G. A., Comerford J. M., 2015, Astrophys. J., 811, 14

McGurk R. C., Max C. E., Rosario D. J., Shields G. A., Smith K. L., Wright S. A., 2011, Astrophys. J. Lett., 738, L2

McMullin J. P., Waters B., Schiebel D., Young W., Golap K., 2007, in Astronomical Society of the Pacific Conference Series, Vol. 376, Astronomical Data Analysis Software and Systems XVI, Shaw R. A., Hill F., Bell D. J., eds., p. 127

Merritt D., Ekers R. D., 2002, Science, 297, 1310

Merritt D., Milosavljević M., 2005, Living Reviews in Relativity, 8

Mezcua M., Lobanov A. P., Chavushyan V. H., LeónTavares J., 2011, Astron. Astrophys., 527, A38

Müller-Sánchez F., Comerford J. M., Nevin R., Barrows R. S., Cooper M. C., Greene J. E., 2015, Astrophys. J., 813, 103

Müller-Sánchez F., Prieto M. A., Hicks E. K. S., VivesArias H., Davies R. I., Malkan M., Tacconi L. J., Genzel R., 2011, Astrophys. J., 739, 69

Murgia M., 2003, Pub. of the Astronomical Society of Aus- 
tralia , 20, 19

Murgia M., Parma P., de Ruiter H. R., Bondi M., Ekers R. D., Fanti R., Fomalont E. B., 2001, Astron. Astrophys., 380, 102

Myers S. T., Spangler S. R., 1985, Astrophys. J., 291, 52

Natarajan P., Pringle J. E., 1998, Astrophys. J. Lett., 506, L97

Noel-Storr J., Baum S. A., Verdoes Kleijn G., van der Marel R. P., O'Dea C. P., de Zeeuw P. T., Carollo C. M., 2003, Astrophys. J. Lett., 148, 419

O'Dea C. P., 1998, Pub. Astron. Soc. Pac., 110, 493

O'Dea C. P., Owen F. N., 1987, Astrophys. J., 316, 95

Pacholczyk A. G., 1970, Radio astrophysics. Nonthermal processes in galactic and extragalactic sources

Parma P., Ekers R. D., Fanti R., 1985, Astron. Astrophys. Suppl., 59, 511

Peck A. B., Taylor G. B., 2001, Astrophys. J. Lett., 554, L147

Peng C. Y., Ho L. C., Impey C. D., Rix H.-W., 2002, Astronom. J., 124, 266

Peng C. Y., Ho L. C., Impey C. D., Rix H.-W., 2010, Astronom. J., 139, 2097

Pringle J. E., 1996, Mon. Not. Roy. Astron. Soc., 281, 357

Pringle J. E., 1997, Mon. Not. Roy. Astron. Soc., 292, 136

Readhead A. C. S., Taylor G. B., Xu W., Pearson T. J., Wilkinson P. N., Polatidis A. G., 1996, Astrophys. J., 460, 612

Rees M. J., 1978, Nature, 275, 516

Rees M. J., Begelman M. C., Blandford R. D., Phinney E. S., 1982, Nature, 295, 17

Rodriguez C., Taylor G. B., Zavala R. T., Peck A. B., Pollack L. K., Romani R. W., 2006, Astrophys. J., 646, 49

Romero G. E., Chajet L., Abraham Z., Fan J. H., 2000, Astron. Astrophys., 360, 57

Roos N., Kaastra J. S., Hummel C. A., 1993, Astrophys. J., 409, 130

Rosario D. J., Shields G. A., Taylor G. B., Salviander S., Smith K. L., 2010, Astrophys. J., 716, 131

Rottmann H., 2001, PhD thesis, Thesis (Ph.D.) - University Bonn, 2001, 180 pages

Salpeter E. E., 1955, Astrophys. J., 121, 161

Sarazin C. L., Begelman M. C., Hatchett S. P., 1980, Astrophys. J. Lett., 238, L129

Sargent W. L. W., 1972, Astrophys. J., 173, 7

Shen Y., Liu X., Greene J. E., Strauss M. A., 2011, Astrophys. J., 735, 48

Sillanpaa A., Haarala S., Valtonen M. J., Sundelius B., Byrd G. G., 1988, Astrophys. J., 325, 628

Smith K. L., Shields G. A., Bonning E. W., McMullen C. C., Rosario D. J., Salviander S., 2010, Astrophys. J., 716,866

Springel V., 2005, Mon. Not. Roy. Astron. Soc., 364, 1105

Steenbrugge K. C., Blundell K. M., 2008, Mon. Not. Roy. Astron. Soc., 388, 1457

Thorne K. S., Braginskii V. B., 1976, Astrophys. J. Lett., 204, L1

Tingay S. J., Wayth R. B., 2011, Astronom. J., 141, 174

Tresse L., Rola C., Hammer F., Stasińska G., Le Fevre O., Lilly S. J., Crampton D., 1996, Mon. Not. Roy. Astron. Soc., 281, 847

Ulvestad J. S., 2003, in Astronomical Society of the Pacific Conference Series, Vol. 300, Radio Astronomy at the
Fringe, Zensus J. A., Cohen M. H., Ros E., eds., p. 97 Valtonen M. J. et al., 2016, Astrophys. J. Lett., 819, L37 van der Laan H., Perola G. C., 1969, Astron. Astrophys., 3, 468

Villata M., Raiteri C. M., 1999, Astron. Astrophys., 347, 30

Wang J.-M., Chen Y.-M., Hu C., Mao W.-M., Zhang S., Bian W.-H., 2009, Astrophys. J. Lett., 705, L76

Whittle M., Rosario D. J., Silverman J. D., Nelson C. H., Wilson A. S., 2005, Astronom. J., 129, 104

Wilkinson P. N., Polatidis A. G., Readhead A. C. S., Xu W., Pearson T. J., 1994, Astrophys. J. Lett., 432, L87

Wirth A., Smarr L., Gallagher J. S., 1982, Astronom. J., 87,602

Xu D., Komossa S., 2009, Astrophys. J. Lett., 705, L20

Zhou H., Wang T., Zhang X., Dong X., Li C., 2004, Astrophys. J. Lett., 604, L33

Zier C., Biermann P. L., 2002, Astron. Astrophys., 396, 91 
Table 1. Sample Galaxy

\begin{tabular}{|l|l|l|}
\hline Parameters & Values & Reference \\
\hline Galaxy name & 2MASX J12032061+1319316 & NED \\
\hline RA & $12: 03: 20.7658$ & NED \\
\hline DEC & $+13: 19: 31.39$ & NED \\
\hline Galaxy Type & S0 & $(\overline{\text { Fathi et al. }}$ 2010). HyperLeda \\
\hline Redshift & 0.058423 & NED \\
\hline Luminosity Distance & $245.4 \pm 17.2 \mathrm{Mpc}$ & NED \\
\hline Radio Loudness Parameter & 156 & $(\overline{\text { Fu et al. }}$ 2012 \\
\hline 1.4 GHz Peak Flux Density & $0.103 \mathrm{Jy} /$ beam & NVSS (beam $\left.=45^{\prime \prime}\right)$ \\
\hline Optical Absolute Mag (z band) & $-22.07 \pm 0.50$ & SDSS DR9 \\
\hline Velocity Dispersion & $189.9 \mathrm{~km} \mathrm{~s}^{-1}$ & pPxf using SDSS DR9 spectra \\
\hline
\end{tabular}

Table 2. Observation Details

\begin{tabular}{|l|l|l|l|}
\hline Frequency & Observing Date & ID & Reference \\
\hline $6 \mathrm{GHz}$ & 20 July, 2015 & VLA/15A-068 & Our Data \\
\hline $8.5 \mathrm{GHz}$ & 14 March, 2014 & VLA/13B-020 & Archival Data \\
\hline $11.5 \mathrm{GHz}$ & 14 March, 2014 & VLA/13B-020 & Archival Data \\
\hline $15 \mathrm{GHz}$ & 29 May, 2016 & VLA/16A-144 & Our Data \\
\hline
\end{tabular}




\section{Rubinur K. et al.}

Table 3. Radio Properties of 2MASXJ12032061+131931

\begin{tabular}{|c|c|c|c|c|c|c|c|c|c|}
\hline & $\begin{array}{l}\text { Array } \\
\text { configuration }\end{array}$ & $\begin{array}{l}\text { Robust } \\
\text { parameter }\end{array}$ & $\begin{array}{l}\text { Image } \\
\text { Noise } \\
\text { in } \mu \mathrm{Jy}\end{array}$ & $\begin{array}{l}\text { Core Size in } \\
\theta_{M} \times \theta_{N}(\prime \prime)\end{array}$ & $\begin{array}{l}\text { Core Peak } \\
\text { Flux in mJy }\end{array}$ & $\begin{array}{l}\text { East Jet } \\
\text { size in /I }\end{array}$ & $\begin{array}{l}\text { South East } \\
\text { Hotspot Peak } \\
\text { Flux in mJy }\end{array}$ & $\begin{array}{l}\text { West Jet } \\
\text { size in } /\end{array}$ & $\begin{array}{l}\text { North West } \\
\text { Hotspot Peak } \\
\text { Flux in mJy }\end{array}$ \\
\hline $6 \mathrm{GHz}$ & $\mathrm{A}$ & -0.5 & 21 & not resolved & - & 1.50 & 11.90 & 1.03 & 8.40 \\
\hline $8.5 \mathrm{GHz}$ & $\mathrm{A}$ & -0.5 & 14 & $24 \times 18$ & 3.8 & 1.11 & 6.86 & 1.20 & 5.68 \\
\hline $8.5 \mathrm{GHz}$ & $\mathrm{A}$ & 0.5 & 17 & Not resolved & - & 1.50 & 8.20 & 1.45 & 6.40 \\
\hline $11.5 \mathrm{GHz}$ & $\mathrm{A}$ & -0.5 & 19 & $20 \times 14$ & 3.2 & 1.05 & 4.90 & 0.96 & 4.44 \\
\hline $15 \mathrm{GHz}$ & $\mathrm{B}$ & -0.5 & 13 & Not resolved & - & 0.90 & 7.30 & 0.90 & 5.00 \\
\hline
\end{tabular}

